\title{
NOESIS: An Enhanced Educational Environment for Kids with Autism Spectrum Disorders
}

\author{
Jason Vittorias, Panagiotis Petrantonakis, Dimitris Bolis, Alexandra Tsiligkyri, \\ Vasiliki Kosmidou, and Leontios J. Hadjileontiadis \\ Dept. of Electrical \& Computer Eng., Aristotle Univ. of Thessaloniki, \\ GR 54124 Thessaloniki Greece \\ leontios@auth.gr
}

\begin{abstract}
A novel educational environment for kids with autistic spectrum disorders (ASDs), namely NOESIS, is presented in this paper. NOESIS takes into account ASD kids' individual characteristics (level of autism, source sensitivity, reaction target, etc), their emotional state (stress level, hyper-/hypo-tension) during their educational procedure, and creativity during guided-and selfactivity (e.g., gaming). It adapts to each kid's specific characteristics through system adaptation and selfregulation procedures. Moreover, it provides assistance to the educator for preparation, customization and optimization of the educational material for each kid and provision of enhanced evaluation procedures (scores/tools) via well-managed Web Services. Parents' updating is also provided via reporting material with learning curve descriptions. Overall, NOESIS contributes to the provision of opportunities to all ASD children to be educated by facilitating access and tuning innovative technology to social needs.
\end{abstract}

\section{Introduction}

Not until the middle of the 20th century was there a name for a disorder that now appears to affect an estimated 3.4 every 1,000 children ages 3-10, a disorder that causes disruption in families and unfulfilled lives for many children. In 1943 Dr. Leo Kanner of the Johns Hopkins Hospital studied a group of 11 children and introduced the label early infantile autism. At the same time a German scientist, Dr. Hans Asperger, described a milder form of the disorder that became known as Asperger syndrome. These two disorders were described as the most frequent pervasive developmental disorders ${ }^{1}$,

\footnotetext{
${ }^{1}$ Rett syndrome and Childhood Disintegrative Disorder are other, quite rare, pervasive developmental disorders.
}

more often referred to today as autism spectrum disorders (ASD). All these disorders are characterized by varying degrees of impairment in communication skills, social interactions, and restricted, repetitive and stereotyped patterns of behavior. From a statistical viewpoint, 1 in 150 children ( 1 in 94 boys) is born with autism, while about 1.5 million Americans have an ASD. Moreover, autism is the fastest-growing developmental disability, with $10 \%$ to $17 \%$ annual growth (one autistic child is born every 20 minutes $)^{2}$. At a financial level, $\$ 90$ billion is the USA annual cost for treatment of autism. Nevertheless, cost of life-long care can be reduced by $2 / 3$ with early diagnosis and intervention. As the central feature of autism is attention-tunnelling, monotropism, computers can be an ideal environment for promoting communication, sociabilility, creativity, and playfulness for individuals even at the extreme of the autistic spectrum. Following the UNESCO principles under the global "Education for All" movement ${ }^{3}$ (www.unesco.org/education), the proposed project, namely NOESIS, aims at normalizing the education procedure of kids with autism spectrum disorders by creating a novel educational tool that takes into account their individual characteristics and adapts the educational procedure accordingly.

\section{NOESIS structure}

NOESIS consists of separate modules that are integrated on a common basis, i.e., to involve kids, educators and parents in an efficient educational environment (see Fig. 1). By implementing the applied behavior analysis (ABA), the most effective education methodology of autistic children [1], enhanced with the latest perspec-

\footnotetext{
${ }^{2}$ Growth comparisons during the 1990s: US population increase $13 \%$; Disabilities increase: $16 \%$; Autism increase: $172 \%$.

${ }^{3}$ UNESCO leads the global Education for All movement, aiming to meet the learning needs of all children, youth and adults by 2015 .
} 


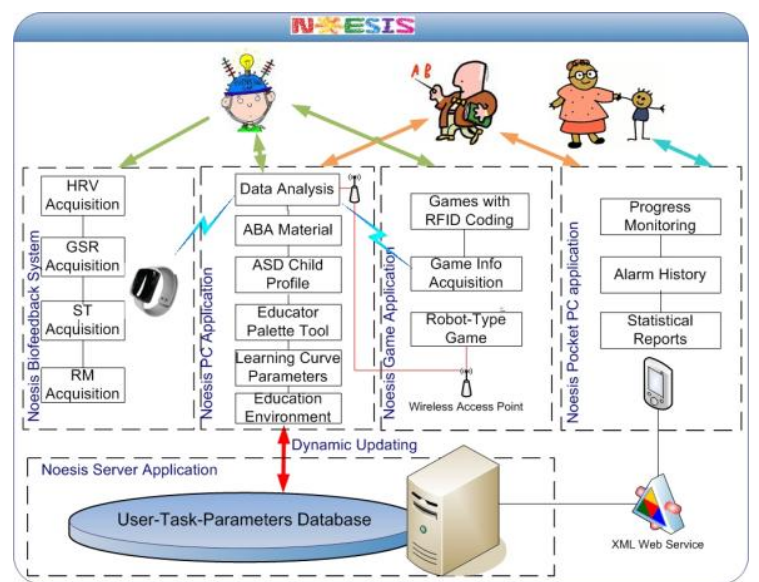

Figure 1. Organizational block-diagram of NOESIS.

tives in autism causes, i.e., embedding educational procedures that aim at mirror neurons (MN) [2] activation, a computer-based tool is designed to assist educators and ASD kids during their education intervention. Noninvasive biofeedback information (such as Heart Rate Variability-HRV and Galvanic Skin Response-GSR, Acceleration-ACC and Skin Temperature-ST) is used to monitor kid's stress during the educational sessions, combined with complexity analysis resulting in adjustable task difficulty, stimuli and help that fit each kid's specific reaction to the current emotional state and task (see Fig. 2).

Transparent monitoring of kid's playing at his/her 'private area', by using Radio-Frequency Identification (RFID) tags-receivers (see Fig. 3) embedded within his/her game parts (e.g., balls, tubes, etc), along with robot-based activities provide additional information about the effects of gaming to his/her educational and social elevation. Moreover, fuzzy logic-based modeling [3] is used across the NOESIS structure to model the whole educational activity, providing real-time scoring information to the educator. With NOESIS, the teacher can select from a variety of 10.000 ABA materials the most appropriate to his/her individual pupil and perform cognitive and meta-cognitive procedures, with regard to the degree of success during the educational intervention (see Fig. 4).

Statistical analysis, comparisons and reports are also outputted from NOESIS, providing valuable information, both to the educator and the parents regarding their kid's progress. NOESIS provides the bed-set not only for exploration of team's abilities to think about and re-design ASD kid's educational approach, but also to understand and integrate the needs of the whole ASD community, in a joint attempt to facilitate their education and contribute to a better quality of life.

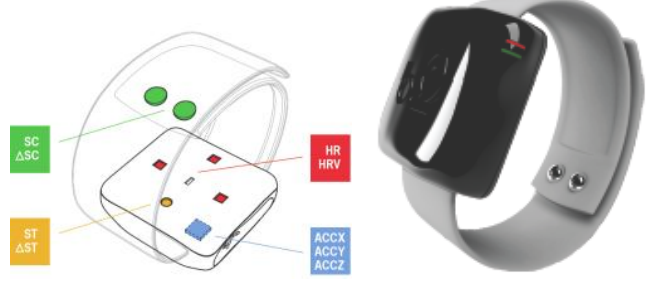

(a)

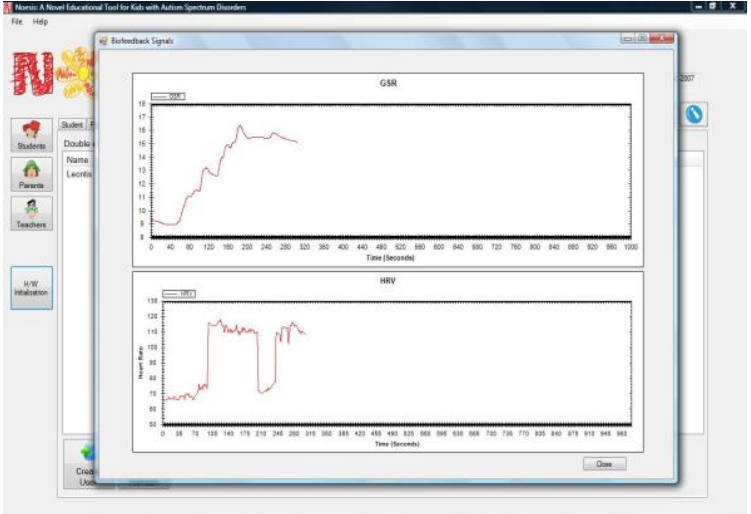

(b)

Figure 2. Monitoring of the biofeedback information of the pupil. (a) The Exmocare BT2 Watch (www.exmocare.com), (b)The monitoring screen of the main biofeedback signals (GSR-top, HRV-bottom).

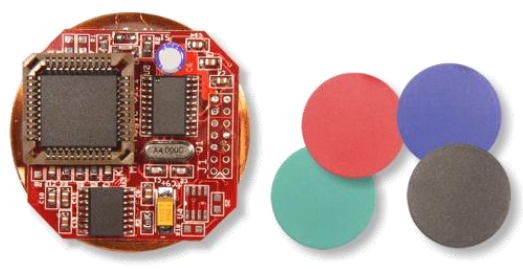

Figure 3. The Mini Magic-232 RFID reader (45 $\times 45 \times 12 \mathrm{~mm}$ ) and the EM4102 RFID tags (3.0

\section{NOESIS user's scenarios}

NOESIS aims at the development of a novel educational tool that will facilitate the learning process of ASD kids and contribute to their sociability, according to a series of scenarios. Let's meet Jim, who will guide us throughout the user scenarios.

\subsection{Scenario \#1: Setting up NOESIS}

Jim is an educator for kids with ASD for 7 years at a special school in Thessaloniki, Greece. His experience 


\section{$\$$
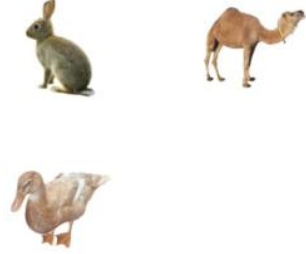 \\ Show lion}

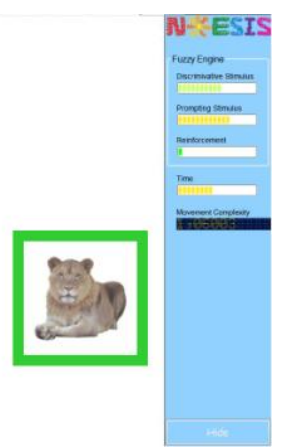

(a)

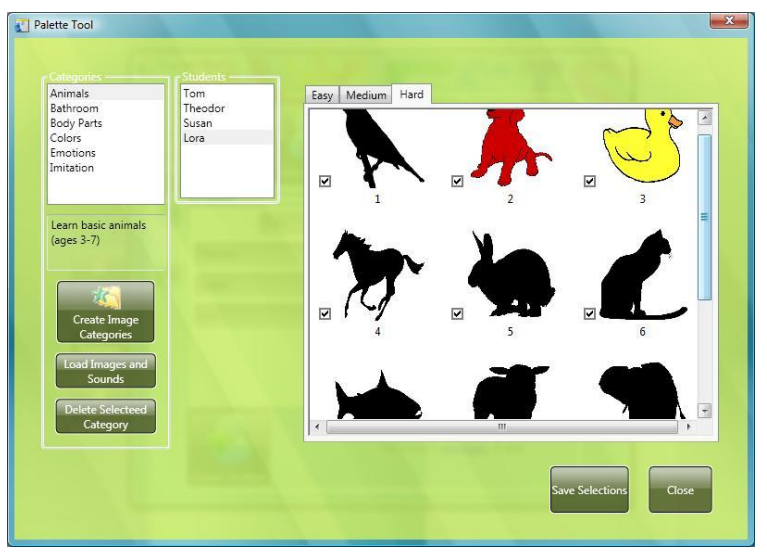

(b)

Figure 4. (a) The ABA-based previewing of the task "Learning the animals", (b) the educator palette tool.

reveals that autistic kids react positively to computer tasks. Lately, he has heard about a new educational tool, namely NOESIS and decides to test its effectiveness. Starting with NOESIS, he chooses which server he prefers to connect to and follows the system instructions for setting up a new account. Adding the pupils that will be attached to his account, he is requested to fill all the parameters that fully describe the autism level and the cognitive age of each individual kid. In this way the system will point out to Jim which tasks best fit to the pupils' needs. Completing the setup the server updates the database with Jim's account. Jim is willing to check it out with one of his pupils, Theodor.

\subsection{Scenario \#2: Learning the animals}

Theodor is a 3 years old boy, who loves the animals, so Jim asks him to play with the "Learning the animals" task. NOESIS adapts the difficulty of the task, the kind and frequency of help and reinforcement according to Theodor's autism parameters that have been setup by Jim. Jim helps Theodor wear the Exmocare BT2 wrist-

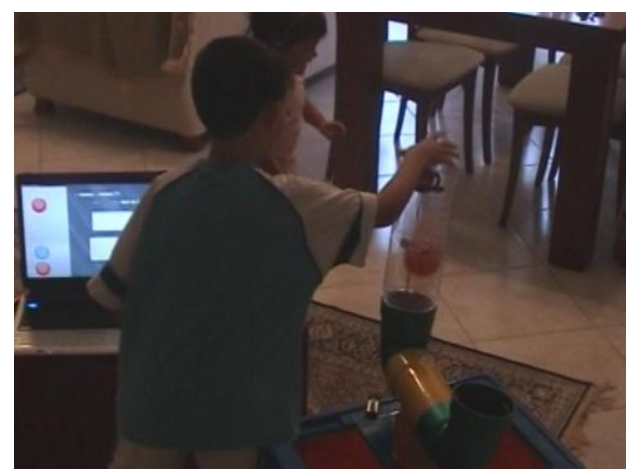

Figure 5. The kid plays the game 'Tubes and Balls' and NOESIS recognizes the color of the ball he is throwing into the tube using RFID coding. A complexity analysis is carried to identify pattern-locking and comprehension of the colors.

watch to enable the biofeedback with the application. The task begins, and a set of animal pictures appears on the screen. Theodor is asked with audio and text commands to show a specific animal through a set of animals. Using the special joystick Theodor replies to questions with great enthusiasm for his new "game" and get a variety of rewards (visual, auditory) from the computer for his learning efforts. After a while, the questions appear to be difficult for Theodor, making him anxious and unwilling to proceed. His anxiety is obvious not only through his difficulty in answering the questions but through the biofeedback monitoring signals, i.e., a sudden raise of the GSR, HRV, ACC and ST parameters, as well. NOESIS sets an alarm to notify Jim, so that he can give additional help to Theodor. This alarm activates the fuzzy engine in order to better adjust to Theodor's personal capabilities. After Theodor completes a session, all the details are stored in the SQL server forming Theodor's personalized log files.

\subsection{Scenario \#3: Enjoying NOESIS}

Break Time! Theodor is in the play room with various toys. Jim reinforces him to play with balls and tubes and leaves the room. Theodor puts balls of different color in the suggested tubes (see Fig. 5). With the help of RFID technology, a PC at the other end of the room simulates his actions online. Jim is careful not to disturb Theodor, so, being at his desk-room, he uses his Pocket PC, connects via Web Services to the server and asks to see the information about Theodor's actions. The amount of the alarms set by NOESIS reveals Theodor's difficulty in distinguishing the colors, making obvious the need for 


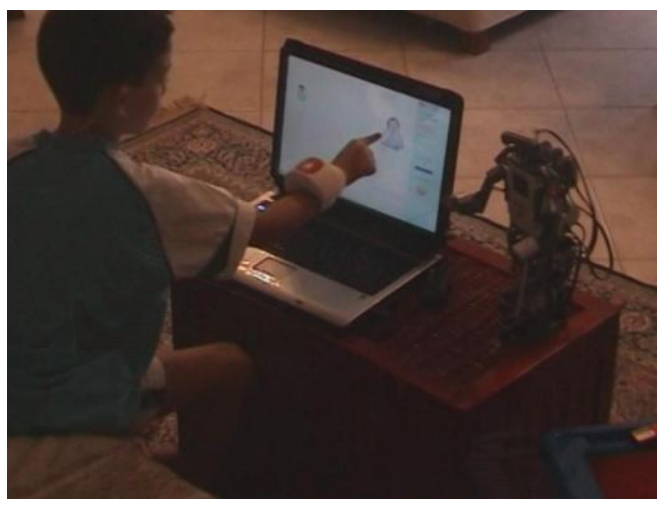

Figure 6. An example of kid's interaction with NOESIS and NOE-robot. Note the Exmocare BT2 Watch in kid's right hand, which does not prevent him to interact.

further training; hence, Jim plans to repeat the related computer task about colors with Theodor.

\subsection{Scenario\#4: Happy collaboration}

Theodor had a great time with the balls, but started complaining because he wanted something more active. Jim comes up with another feature of NOESIS, RoboNOESIS - a wireless robot, namely 'NOE'. Theodor immediately is fully concentrated to his new "friend". NOE is used as a rewarding means that reacts as Theodors interacts with the tasks of NOESIS, and tries to elevate Theodor's sociality, eliminating at the same time any obligations for social demands (see Fig. 6). NOE gives audio-kinetic feedback to Theodor and when the latter is inactive for more than 10 seconds, NOE takes the initiative to "provoke" Theodor to react and participate in the educational process.

\subsection{Scenario \#5: Evaluating the Day}

Jim is at home and decides to review Theodor's progress. Using his Pocket PC, he downloads all Theodor's data that are available to the NOESIS server via Web Services (see Fig. 7(a)). Jim has access to all the sessions, being able to review each alarm. Choosing to compare the sessions that Theodor performed with the ball game, a comparison graph is generated, showing a slight progress at the learning abilities of Theodor across successive sessions. Viewing the patterns that Theodor created so far, Jim can decide about Theodor's creativity and imagination or repetition. Focusing on the log files of the "Learning the animals" task, Jim observes that a certain image, the image of a tiger, makes Theodor ex-

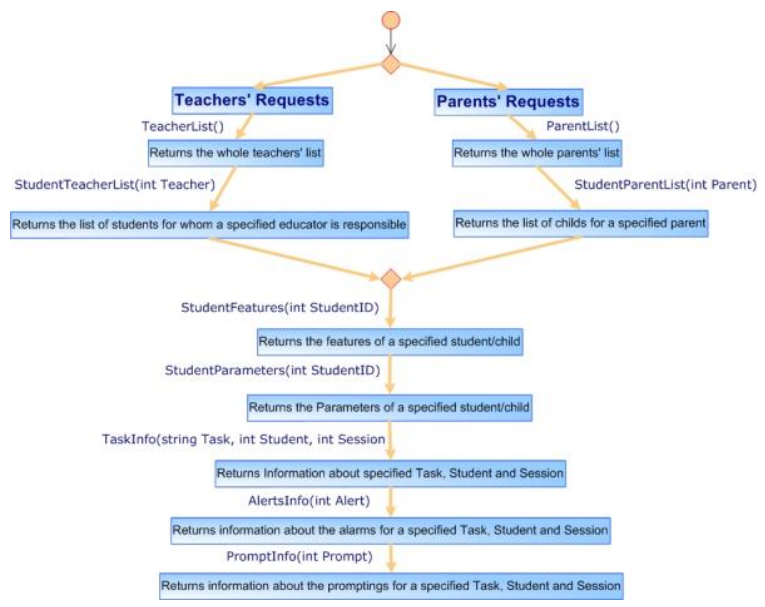

(a)
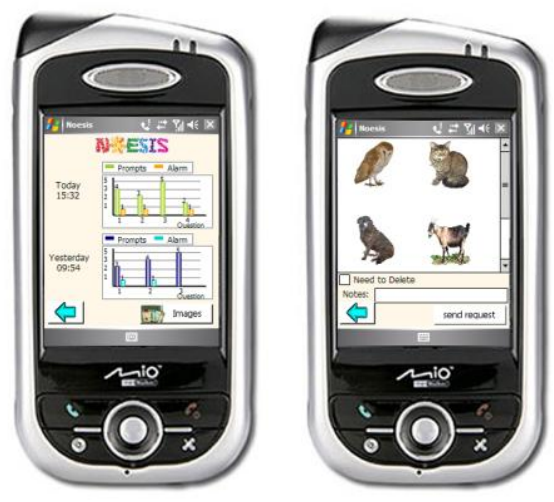

(b)

Figure7(a). Flow-chart of the Web servicesbased retrieval information of teachers and/or parents from the server, (b) Review of kid's alarm statistics and handling of the customization of the ABA material through a Pocket PC via Web services.

tremely anxious and loses his concentration. So, Jim decides to send a request of deleting this image from Theodors sessions, via Web services (see Fig. 7(b)).

Moreover, Jim has also the opportunity to monitor his class (either on- or off-line) through the class supervision tool of NOESIS (see Fig. 8).

\subsection{Scenario \#6: Involving Parents}

Theodor's father, Bill, is at work thinking about how his son is doing at school; hence, he connects to the NOESIS server as a parent-user and asks for Theodor's updated reports. In a few seconds, he previews a history of Theodor's learning progress scores and alarm series during the current month, along with the current status of 


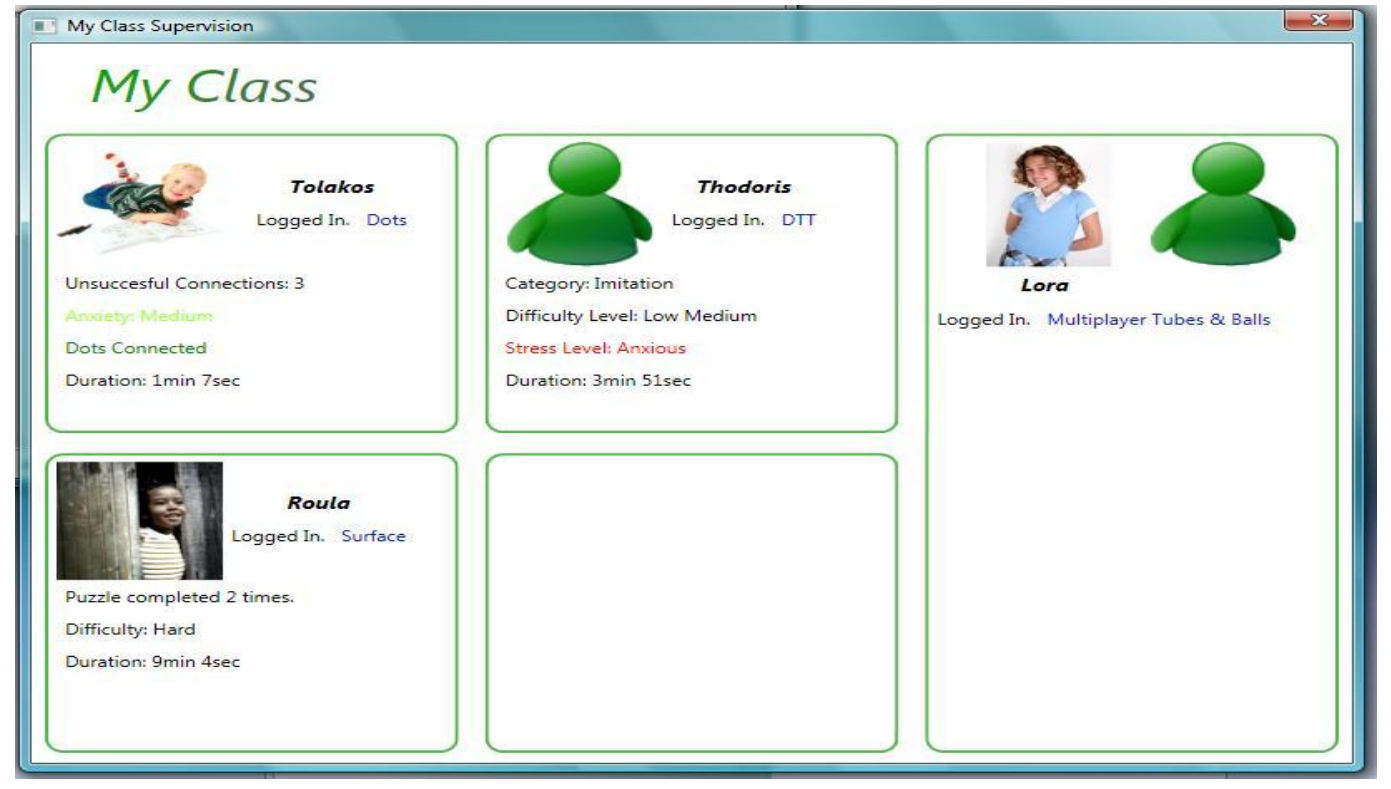

Figure 8. The class supervision tool of NOESIS.

Theodor's basic educational sections, i.e., cognitive, mobility, verbal ability, communication-imagination (see Fig. 9). Bill reinforces Jim to keep involving NOESIS in the educational procedures of Theodor.

Through the NOESIS 'Listen to the parents' voice' common space, Bill has also the opportunity to share his views, thoughts and case-scenarios with other parents and discuss and send feedback to Jim upon specific issues and decisions.

\section{Concluding remarks}

The innovations behind NOESIS consist of a broader approach to the problem examined. Until now, ASD software, like BoardMaker [4] and Picture It [5], refers to educational material gathering. Using advanced signal processing and cutting-edge technology NOESIS provides an integrated educational environment which is closer to the kid's needs and, therefore, realistic and more efficient. It is the first ASD educational tool, which uses both biofeedback information and kid's response in an adaptive way that fulfills each kid's specific educational needs.

NOESIS provides the educator with flexible ways of setting, evaluating and expanding his/her educational material, combining information from the kid's real world, capturing the essence of his/her understanding abilities and adjusting his/her learning rhythm, under a vast variety of different case-study scenarios.

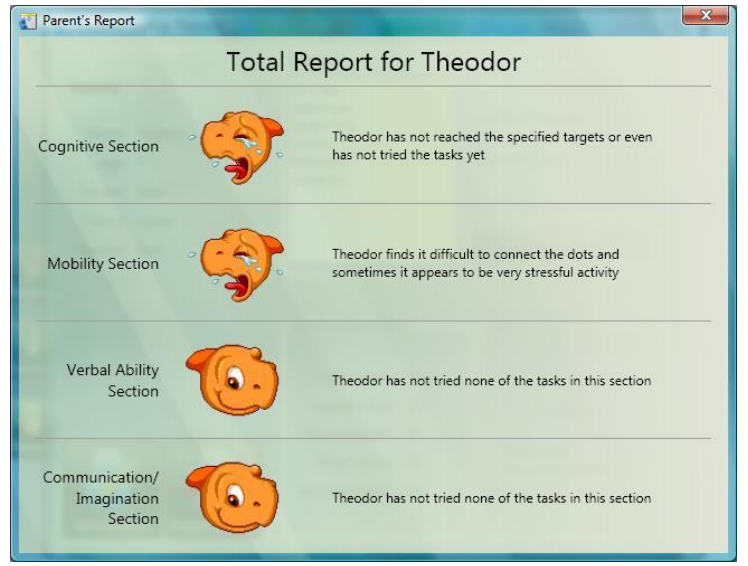

\section{Figure 9. Parent's report for four basic sec- tions of kid's activities.}

\section{References}

[1] O.I. Lovaas, "Behavioral treatment and normal educational and intellectual functioning in young autistic children", $J l$ of Cons. \& Clinical Psychology, vol. 55, 1989, pp. 3-9.

[2] L.M. Oberman, E.M. Hubbard, J.P. McCleery, E.L. Altschuler, J.A. Pineda, and S. Vilayanur, "EEG evidence for mirror neuron dysfunction in autism spectrum disorders," Cognitive Brain Research, vol. 24, 2005, pp. 190-198.

[3] Zadeh, L.A., Fuzzy sets, in: Dubois, Prade, Yager (Eds.), Readings in Fuzzy Sets for Intelligent Systems, Morgan Kaufmann Publishers, Inc. Los Altos, CA, , pp. 27-65, 1993.

[4] http://www.mayer-johnson.com:80/

[5] http://www.slatersoftware.com/autism.html 